\title{
MRI assessment of cortical thickness and functional activity changes in adolescent girls following three months of practice on a visual-spatial task
} Richard J Haier*1, Sherif Karama², Leonard Leyba ${ }^{3}$ and Rex E Jung3,4

Address: ${ }^{1}$ School of Medicine (Emeritus), University of California, Irvine CA, USA, ${ }^{2}$ McConnell Brain Imaging Center, Montreal Neurological Institute, McGill University, Montreal, Canada, ${ }^{3}$ Mind Research Network, Albuquerque, New Mexico, USA and ${ }^{4}$ Department of Neurosurgery, University of New Mexico, Albuquerque, New Mexico, USA

Email: Richard J Haier* - rich.haier@gmail.com; Sherif Karama - sherifkarama@gmail.com; Leonard Leyba - lleyba@mrn.org; Rex E Jung - rex.jung@gmail.com

* Corresponding author

Published: I September 2009

BMC Research Notes 2009, 2:174 doi:10.1 186/1756-0500-2-174
Received: 14 April 2009

Accepted: I September 2009

This article is available from: http://www.biomedcentral.com/1756-0500/2//74

(c) 2009 Haier et al; licensee BioMed Central Ltd.

This is an Open Access article distributed under the terms of the Creative Commons Attribution License (http://creativecommons.org/licenses/by/2.0), which permits unrestricted use, distribution, and reproduction in any medium, provided the original work is properly cited.

\begin{abstract}
Background: Neuro-imaging studies demonstrate plasticity of cortical gray matter before and after practice for some motor and cognitive tasks in adults. Other imaging studies show functional changes after practice, but there is not yet direct evidence of how structural and functional changes may be related. A fundamental question is whether they occur at the same cortical sites, adjacent sites, or sites in other parts of a network.
\end{abstract}

Findings: Using a 3 T MRI, we obtained structural and functional images in adolescent girls before and after practice on a visual-spatial problem-solving computer game, Tetris. After three months of practice, compared to the structural scans of controls, the group with Tetris practice showed thicker cortex, primarily in two areas: left BAs 6 and 22/38. Based on fMRI BOLD signals, the Tetris group showed cortical activations throughout the brain while playing Tetris, but significant BOLD decreases, mostly in frontal areas, were observed after practice. None of these BOLD decreases, however, overlapped with the cortical thickness changes.

Conclusion: Regional cortical thickness changes were observed after three months of Tetris practice. Over the same period, brain activity decreases were observed in several other areas. These data indicate that structural change in one brain area does not necessarily result in functional change in the same location, at least on the levels assessed with these MRI methods.

\section{Findings}

Plasticity of gray matter (GM) in adults has been demonstrated before and after practice of both motor and cognitive tasks [1-3]. Evidence points to plasticity effects for both novel learning of a task and for continued practice of a task once learned [4]. A fundamental question is whether GM increases after practice in a specific brain area are concomitant with functional changes in the same area (site specific), and/or whether different areas in the same network show changes. A secondary question is whether GM increases are associated with increased or decreased brain activity. Functional imaging studies show both directions [5-11]. With two exceptions, none of these included structural assessments so any overlap of functional and structural changes could not be determined. One exception [12] reported limited overlap for a mirror 
reading task; the other [13] reported no overlap for a simple visual-motor task.

We conducted a straightforward test of the site-specific hypothesis. We first examined whether cortical thickness (CT) increased after three months of practicing a visualspatial game, Tetris. Then, in the same subjects, we determined blood oxygen level dependent (BOLD) changes after practice. Finally, we examined the overlap of the structural and functional results. Since Tetris involves rapid visual-spatial rotation problem-solving and motor co-ordination, we hypothesized GM increases in premotor and post-central gyri and parts of the parietal and occipital lobes as shown in previous studies [5,14-16].

\section{Methods}

\section{Ethics Statement}

Each participant gave written assent and a parent gave written informed consent as approved by the University of New Mexico Institutional Review Board. This research was conducted in accord with the Helsinki Declaration.

\section{Subjects and Procedure}

Because developing brains are most likely to show potential changes, we studied adolescents. We chose girls to minimize bias based on previous video game experience.

Twenty-six girls aged 12-15 were recruited from advertisements and visits to schools. All were familiar with computers but none reported significant interest in computer game play (defined as playing any games requiring rapid visual-spatial puzzle solving on a regular basis) or significant exposure to Tetris (defined as playing more than one time, or watching someone else play more than three times). None had medical illness, brain injury, psychiatric history or were taking prescription medications. Girls were randomly assigned to either the Tetris practice group $(\mathrm{n}=15)$ or the control group $(\mathrm{n}=11)$. The groups did not differ on age (mean $13.1+/-1.1$ vs. $12.9+/-1.0$, respectively, $\mathrm{t}=.51, \mathrm{NS})$, or FSIQ $(113.8+/-13.4$ vs. $115.7+/-$ $11.4, \mathrm{t}=.40, \mathrm{NS})$.

The Tetris group got online access to a controlled web site where they logged in to play. Each login was tracked for
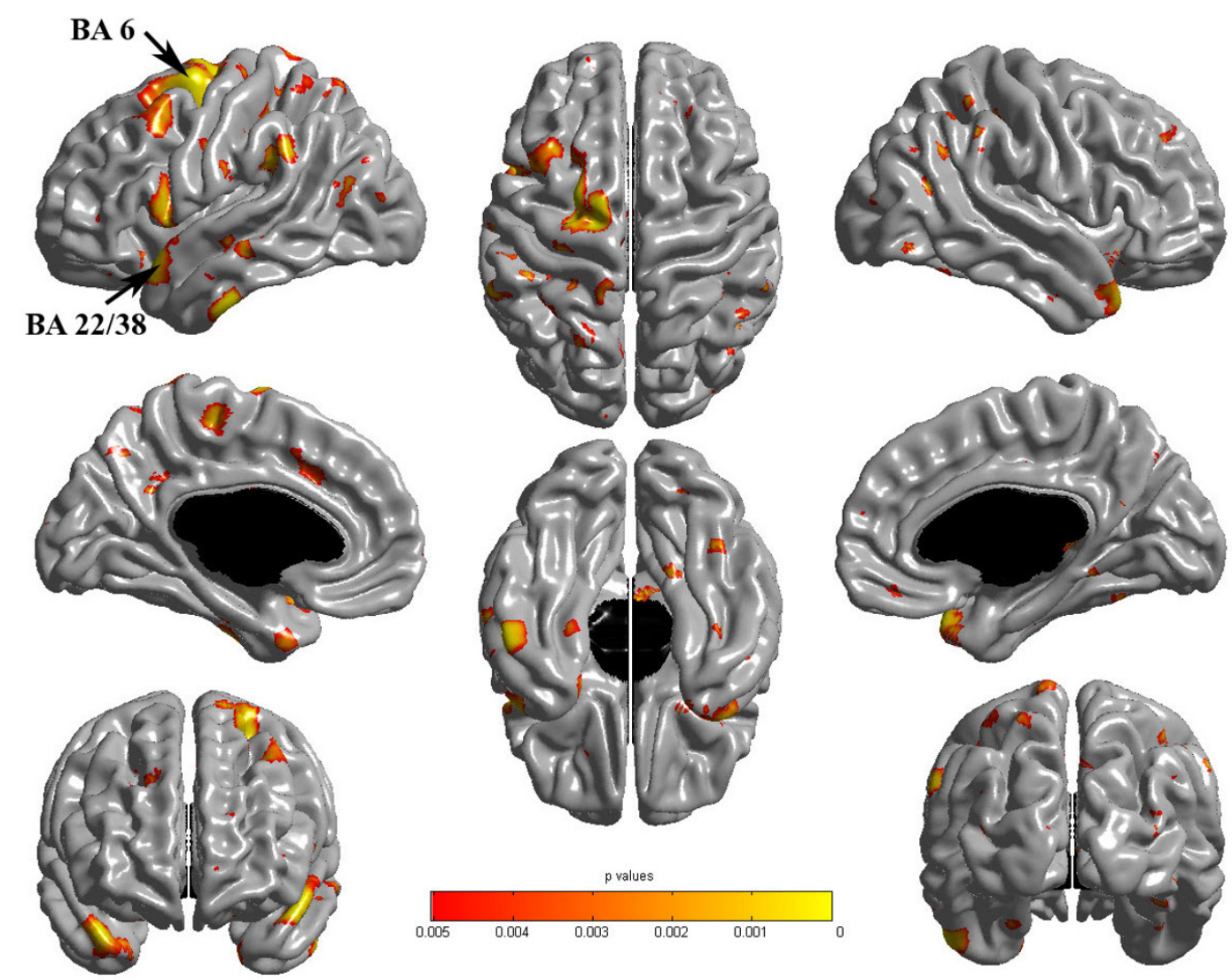

\section{Figure I}

Cortical Thickness Changes. Cortical thickness changes after practice showing Tetris follow-up minus baseline versus Controls follow-up minus baseline, $\mathrm{p}<.005$. Upper left is left hemisphere; arrows show cluster level $\mathrm{p}<.05 \mathrm{FWE}$ corrected. 


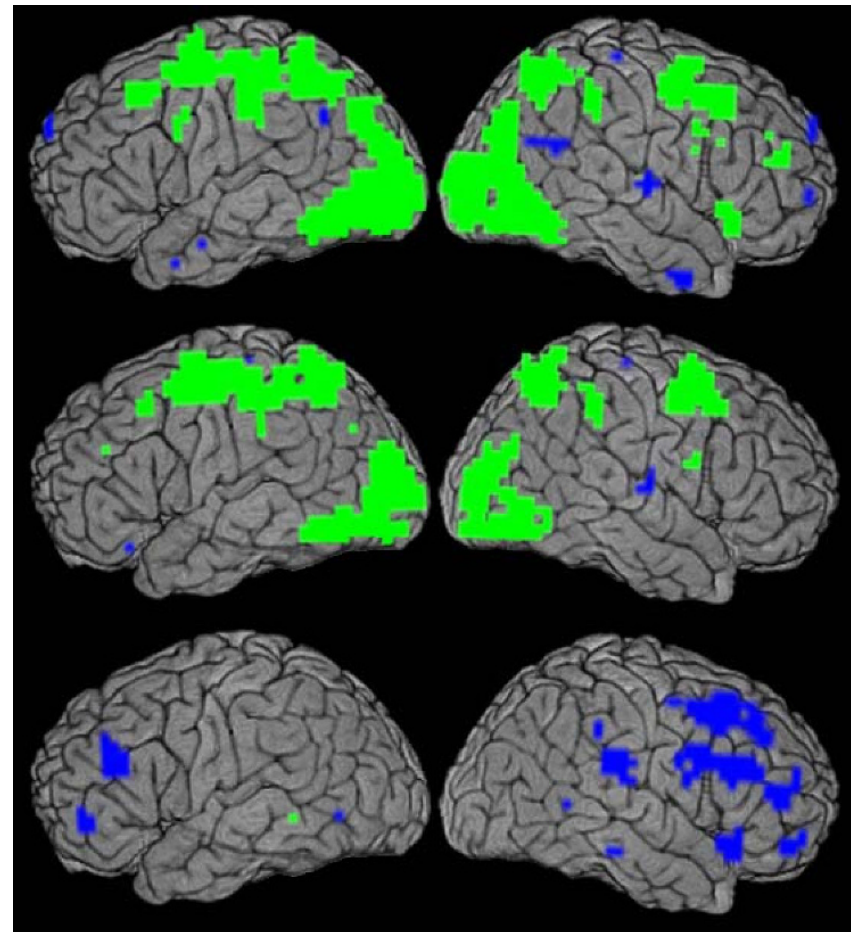

Figure 2

BOLD Signal Changes. BOLD signal increases (green) and decreases (blue) in cortex during the Tetris task (top row is at baseline; middle row is at follow-up; $p<.05$, FWE) and after practice (follow-up minus baseline; bottom row; $p<$ .05 , FDR). Left column is left hemisphere. Template is ICBM152.

duration and scores. Each girl received Tetris instruction and 15 minutes of practice when they entered the study. They were instructed to practice as their schedules permitted during a three-month period. On average, the girls practiced 1.5 hours per week (individual games take only a few minutes). The Control group had no access to the Tetris site and was instructed not to play Tetris during the three-month period. Both groups had a structural MRI at the start and end of the study. Both groups also had fMRI scans, while playing Tetris, at both MRI sessions. Before beginning fMRI, both groups received Tetris instructions and practiced 15 minutes.

\section{Tetris Task}

In random order, pieces with different shapes constructed from 4 squares, appear one at a time at the top of the computer screen. As the piece falls to the bottom, the player uses four buttons to move and/or rotate the piece so it fits in with previous pieces to form rows with no gaps. When a row is completed, it disappears from the screen, and all the remaining pieces drop down, changing the configuration of the play space. This requires rapid analysis of the best way to place each piece into the existing pattern. The version used here was scored as time it took to complete 40 rows.

\section{MRI Acquisition}

Images were obtained on a 3 T Siemens TrioTim scanner. Structural (sMRI) images were collected using a 5 echo mprage sequence $[\mathrm{TE} 1=1.64 \mathrm{~ms}$, TE2 $=3.5 \mathrm{~ms}$, TE3 = $5.36 \mathrm{~ms}$, TE4 $=7.22 \mathrm{~ms}$, TE5 $=9.08 \mathrm{~ms}$; $\mathrm{TR}=2530 \mathrm{~ms}$; $\mathrm{FOV}=256 \mathrm{~mm}$; total acquisition time $=6: 03] .1923 \mathrm{D} 1$ $\mathrm{mm}$ thick slices ( $0 \mathrm{~mm}$ skip) were selected to provide coverage of the entire brain (voxel size: $1 \mathrm{~mm} \times 1 \mathrm{~mm} \times 1$ $\mathrm{mm}$ ). Functional echo planar (fMRI) images were collected using a single-shot sequence $[\mathrm{TE}=29 \mathrm{~ms} ; \mathrm{TR}=2000$ ms; FOV $=24 \mathrm{~cm}$; matrix size $=64 \times 64 ; 32$ axial $3.5 \mathrm{~mm}$ slices (1.05 mm skip); voxel size: $3.75 \times 3.75 \times 4.55$ ].

\section{MR Image Processing}

\section{Cortical thickness}

Structural images were submitted to the CIVET pipeline (1.1.9) http://wiki.bic.mni.mcgill.ca/index.php/CIVET for fully automated analysis. Steps, detailed elsewhere [17], include: 1) Registering to MNI space. 2) Producing high-resolution hemispheric surfaces with 40962 vertices each. 3) Registering surfaces to a high-resolution template. 4) Applying a reverse of step 1 allowing CT estimations in native space of each subject. 5) Smoothing using 20-milimeter kernel [18]. 6) Calculating CT at each vertex.

\section{Brain function}

Subjects alternated between playing Tetris (ON) for 30 seconds using an MRI compatible device and keeping their eyes fixed (OFF) on a (+) sign for 20 seconds. Each run consisted of $11 \mathrm{ON} / \mathrm{OFF}$ cycles totalling 9 minutes 10 seconds per run. On both visits, subjects performed two runs, averaged together for analyses. fMRI images were motion corrected, and normalized to the MNI stereotaxic space using SPM http://www.fil.ion.ucl.ac.uk and re-sampled to $4 \times 4 \times 4 \mathrm{~mm}$. Data for which motion parameters deviated $>3$ S.D. from the mean were excluded leaving 11 Tetris and 9 control subjects. Deconvolution determined the hemodynamic response function (HRF) corresponding to the 50-second ON/OFF period and was converted to percent signal change versus baseline. Empirical examination of this 50 second $\mathrm{HRF}$ in responsive regions showed large percent signal changes from 4 to 36 seconds into the ON/OFF period. The mean percent signal change from 4-36 seconds, encompassing the theoretical peak of the hemodynamic response [19], was used as a single measure of percent signal change per voxel.

\section{Statistical Analyses \\ sMRI}

Analyses used SurfStat created for MATLAB 7 (The MathWorks, Inc.). Baseline cortical thickness maps were subtracted, for each subject, from the 3-month cortical thickness maps, yielding subject-specific maps of change 
Table I: Peak vertex coordinates (MNI) and brain areas where change over three months showed greater cortical thickness in the Tetris group than in the controls $(p<0.005$, uncorrected; $* p<.05$ cluster level, FWE corrected).

\begin{tabular}{|c|c|c|c|}
\hline Brodmann Area & Region Name & $\mathbf{X}, \mathbf{Y}, \mathbf{Z}$ Coordinates & P Value \\
\hline \multicolumn{4}{|l|}{ Left Frontal } \\
\hline BA 6 & Superior frontal gyrus & $-13,-10,69$ & 0.00004 \\
\hline BA 6 & Superior frontal gyrus & $-24,0,67$ & $0.00007^{*}$ \\
\hline BA 6 & Superior frontal gyrus & $-15,-4,74$ & 0.0001 \\
\hline BA 6,8 & Middle frontal gyrus & $-44,14,45$ & 0.0013 \\
\hline BA 6,44 & Inferior frontal gyrus & $-52,12,10$ & 0.0005 \\
\hline BA 45,46 & Inferior frontal gyrus & $-4 I, 4 I, 5$ & 0.004 \\
\hline \multicolumn{4}{|l|}{ Right Frontal } \\
\hline BA9 9 & Superior frontal gyrus & $20,39,39$ & 0.002 \\
\hline \multicolumn{4}{|l|}{ Left Parietal } \\
\hline BA 7 & Superior parietal lobule & $-36,-5 I, 64$ & 0.003 \\
\hline BA 7 & Superior parietal lobule & $-24,-67,63$ & 0.002 \\
\hline BA 7 & Superior parietal lobule & $-13,-45,76$ & 0.002 \\
\hline BA 5 & Superior parietal lobule & $-25,-41,60$ & 0.002 \\
\hline BA 19,39 & TemporoParietoOccipital junction & $-44,-71,19$ & 0.002 \\
\hline BA 40 & Supramarginal gyrus & $-63,-42,46$ & 0.0004 \\
\hline BA 31 & Precuneus & $-5,-64,45$ & 0.002 \\
\hline BA 31 & Precuneus & $-10,-53,29$ & 0.003 \\
\hline BA 4 & Paracentral Lobule & $-7,-25,61$ & 0.0001 \\
\hline \multicolumn{4}{|l|}{ Right Parietal } \\
\hline $\mathrm{BA} 40$ & Supramarginal gyrus & $6 I,-4 I, 43$ & 0.0002 \\
\hline BA 39 & Angular gyrus & $47,-57,34$ & 0.001 \\
\hline BA 39 & Angular gyrus & $50,-52,49$ & 0.004 \\
\hline BA 7 & Superior parietal lobule & $4 I,-47,55$ & 0.0009 \\
\hline \multicolumn{4}{|l|}{ Left Temporal } \\
\hline BA $22 / 38$ & Anterior superior temporal gyrus & $-50,16,-16$ & $0.00008^{*}$ \\
\hline BA 20 & Anterior Inferior temporal gyrus & $-58,-15,-35$ & 0.0002 \\
\hline BA 21 & Middle temporal gyrus & $-65,-25,-12$ & 0.001 \\
\hline BA 38 & Medial temporal pole & $-22,7,-38$ & 0.002 \\
\hline BA 28 & Parahippocampal gyrus & $-28,-20,-29$ & 0.002 \\
\hline \multicolumn{4}{|l|}{ Right Temporal } \\
\hline BA 38 & Temporal pole & $41,22,-29$ & 0.0004 \\
\hline BA 37 & Posterior inferior temporal gyrus & $39,-58,-20$ & 0.002 \\
\hline BA 37 & Posterior inferior temporal gyrus & $45,-72,-9$ & 0.0008 \\
\hline BA 36 & Lingual gyrus & $19,-43,-10$ & 0.001 \\
\hline \multicolumn{4}{|l|}{ Left Occipital } \\
\hline BA 19 & Lateral occipital gyrus & $-40,-85,11$ & 0.003 \\
\hline \multicolumn{4}{|l|}{ Right Occipital } \\
\hline $\mathrm{BA} 19$ & Lateral occipital gyrus & $37,-88,14$ & 0.002 \\
\hline \multicolumn{4}{|l|}{ Left Cingulate } \\
\hline BA 24 & Anterior cingulate gyrus & $-9,19,38$ & 0.004 \\
\hline BA 23, 31 & Posterior cingulate gyrus & $-4,-38,27$ & 0.001 \\
\hline
\end{tabular}

in cortical thickness. These maps for the Tetris group were compared to those for the Control group. Results are shown at $\mathrm{p}<.05$ using the Family Wise Error (FWE) correction (Figure 1 ); trends are noted at $\mathrm{p}<.005$, uncorrected. For all tables, Brodmann areas (BA) were determined as best estimates based on the Talairach and Tourneau atlas [20,21].

\section{fMRI}

Individual subject voxel-wise percent BOLD signal change in response to Tetris was passed on to level 2 group analysis. One sample t-tests ( $\mathrm{df}=10$ for Tetris group, $\mathrm{df}=8$ for controls, SPM) against the null hypothesis showed areas significantly modulated by Tetris ( $\mathrm{p}<0.05$; Figure 2, top two panels). Practice effects in the Tetris group were assessed using a paired two-sample t-test contrasting the follow-up vs. baseline visits (Figure 2, lower panel). Group-by-visit interactions were assessed using an unpaired t-test $(\mathrm{df}=18)$ contrasting (Percent signal change at 3 months - Baseline Percent signal change in Experimental Group) Minus (Percent signal change at 3 months - Baseline Percent signal change in Experimental Group). Stereotaxic coordinates of peak statistical significance were converted from ICBM-152 to Talairach compatible coordinates [21]. 
Table 2: Brain areas with decreases in BOLD signal after practice (follow-up minus baseline; $p<.05$ FDR).

\begin{tabular}{|c|c|c|c|}
\hline Brodmann Area & Region Name & X, Y, Z Coordinates (MNI) & P Value \\
\hline \multicolumn{4}{|l|}{ Left Frontal } \\
\hline BA 9 & Middle Frontal Gyrus & $-38,30,26$ & 0.031 \\
\hline BA 46 & Inferior Frontal Gyrus & $-46,42,-2$ & 0.038 \\
\hline \multicolumn{4}{|l|}{ Right Frontal } \\
\hline BA 32 & Medial Frontal Gyrus & $10,18,46$ & 0.026 \\
\hline BA 8 & Superior Frontal Gyrus & $6,34,46$ & 0.026 \\
\hline BA 6 & Middle Frontal Gyrus & $42,6,50$ & 0.031 \\
\hline BA 46 & Middle Frontal Gyrus & $50,42,14$ & 0.032 \\
\hline BA 9 & Middle Frontal Gyrus & $50,26,26$ & 0.033 \\
\hline BA 6 & Precentral Gyrus & $58,6,34$ & 0.035 \\
\hline BA 47 & Inferior Frontal Gyrus & $38,22,-14$ & 0.033 \\
\hline$*$ & Sub-Gyral & $38,50,-10$ & 0.033 \\
\hline BA 6 & Medial Frontal Gyrus & $6,-6,54$ & 0.039 \\
\hline BA 6 & Precentral Gyrus & $38,2,30$ & 0.046 \\
\hline \multicolumn{4}{|l|}{ Right Parietal } \\
\hline BA 40 & Inferior Parietal Lobule & $66,-30,26$ & 0.026 \\
\hline BA 40 & Sub-Gyral & $38,-38,38$ & 0.043 \\
\hline BA 40 & Inferior Parietal Lobule & $58,-38,38$ & 0.049 \\
\hline BA 40 & Postcentral Gyrus & $58,-22,18$ & 0.05 \\
\hline \multicolumn{4}{|l|}{ Left Occipital } \\
\hline BA 19 & Middle Occipital Gyrus & $-58,-66,-2$ & 0.038 \\
\hline \multicolumn{4}{|l|}{ Right Temporal } \\
\hline BA 22 & Superior Temporal Gyrus & $50,-26,-14$ & 0.044 \\
\hline BA 22 & Superior Temporal Gyrus & $58,-50,6$ & 0.049 \\
\hline \multicolumn{4}{|l|}{ Right Cingulate } \\
\hline BA 24 & Cingulate Gyrus & $10,14,30$ & 0.038 \\
\hline BA 24 & Anterior Cingulate & $10,30,22$ & 0.038 \\
\hline
\end{tabular}

We limit discussion to the more conservative FWE results since the chance of Type I error is greater for results based on more lenient threshold methods of FDR or uncorrected $\mathrm{p}$ values. Nonetheless, we included these findings in the tables to increase the chance of replication by other studies.

\section{Results}

Changes in CT following practice led to a significantly thicker cortex in the experimental compared to the control group [Tetris follow-up minus baseline] minus [control follow-up minus baseline], in two Brodmann areas (BA), left BA 6 and left BA 22/38, (p<.05, FWE corrected); these are shown in Figure 1 along with other areas where there was a trend of increases after practice $(\mathrm{p}<.005$, uncorrected). These areas were distributed throughout the brain (Table 1) and were most significant $(\mathrm{p}<.0005$, uncorrected) in BA 6 and 44 (left frontal lobe), BA 40 (bilateral parietal lobe), BA 4 (left paracentral lobule), BA 20 and 22 (left temporal lobe), and BA 38 (right temporal pole). There was no evidence of any areas of thinner cortex after practice.

Tetris-induced BOLD signal increases ( $\mathrm{p}<0.05$, FWE corrected) are similar for both visits (Figure 2, top two panels; Additional Files 1, 2, 3 \&4). Most are in frontal and parietal lobes including parts of BAs $6,7,40$, although the fol- low-up visit showed additional activations in occipital BAs 17,18 , and 19 . For both visits, only a few small clusters (<20 voxels each) showed a significantly decreased BOLD signal during task performance. The same comparisons for the control group showed similar results.

For the Tetris group, no activation changes over the 3month practice period were significant after FWE correction. However, changes based on statistical false discovery rate control (FDR) [22,23] are shown in Figure 2 (lower panel) and Table 2 . These were all decreases after practice and were mostly in the right hemisphere; the most significant were in frontal BAs 32, 8, 9, 6, 46, and in parietal BA 40.

Figure 3 shows both CT change and BOLD signal during Tetris play. For baseline and follow-up, overlap was limited to a few small areas of BAs 6, 7, 22, and 19 (arrows in Figure 3, top and middle rows). The areas with decreased activation over the practice period (follow-up minus baseline) showed no overlap with CT increases (bottom row). To increase statistical power, we did additional analyses, first restricting the CT analyses to regions that showed BOLD changes; and second, restricting the BOLD analyses to regions that showed CT changes. Neither analysis identified any new regions of overlap ( $\mathrm{p}<.005$, uncorrected). 


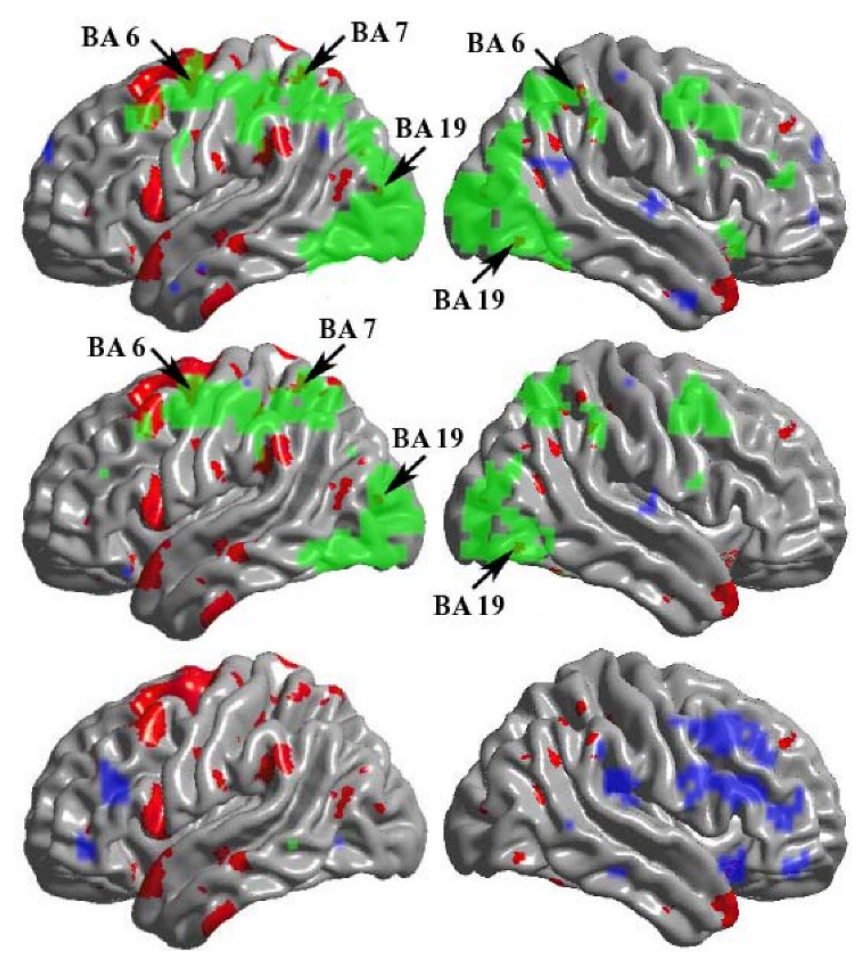

Figure 3

Cortical Thickness and BOLD Changes. Both cortical thickness change after practice (red) and BOLD signal increases (green) or decreases (blue) during Tetris task are shown. Top row is at baseline, middle row is at follow-up, bottom row is follow-up minus baseline. Left column is left hemisphere. Template is Surfstat MNI. Arrows indicate small areas of overlap.

\section{Discussion}

We found no apparent overlap between structural and functional findings to support the site-specific hypothesis. Interpreting fMRI data has important limitations. For example, the BOLD signal reflects neuronal mass activity. It does not distinguish between excitatory and inhibitory activity, and a marked change in processing strategies involving an anatomical area does not necessitate a change in BOLD [24]. It is possible that practiced-subjects developed a more sophisticated approach, but if this did not invoke novel brain areas, it might not be detectable using fMRI.

The frontal eye fields in left BA 6 showed the most significant CT change, consistent with other imaging studies of visual-spatial tasks. The same area also showed increased activity while the subjects played at baseline, but there was no additional change after practice. Notably, BA 6 is at a critical juncture between "intention" and "action" [25], linking memory of previous responses and the rewards associated with them [26], and is active when motor plans are altered [27]. The left temporal pole (BA 22/38) also showed change. The temporal poles integrate visual, auditory, tactile, and internal physiological information, suggesting a critical role in multimodal perceptual analysis [28]. Moreover, there are lateralizing differences, with right more associated with emotion and socially relevant episodic memories, and left associated with semantic memory[29]. We interpret the temporal pole changes as reflecting increased multisensory integration to support visual, spatial, and tactile input necessary to succeed at Tetris. That greater cortical change was seen in the left temporal pole, suggests Tetris play is processed as a general cognitive puzzle rather than a discrete episodic memory. Whether practiced Tetris play and associated cortical changes generalize to performance changes in other cognitive domains (e.g. working memory, processing speed, spatial reasoning) is not yet determined.

\section{Conclusion}

These data indicate that structural and functional brain changes after practice do not necessarily occur in the same location, at least on the levels assessed with these MRI methods.

\section{Competing interests}

This project was funded at the MIND Research Network, Albuquerque, N.M. by Blue Planet Software (BPS), Inc., the company holding exclusive licensing rights to Tetris. REJ was the PI and LL received partial salary support. The funders had no role in study design, data collection and analysis, decision to publish, or preparation of the manuscript. RJH is a paid consultant to Blue Planet Software.

\section{Authors' contributions}

RJH and REJ conceived and designed the experiment. REJ performed the experiment. SK and LL analyzed the data. All authors approved and helped write the manuscript.

\section{Additional material}

\section{Additional file 1}

Brain areas of increased BOLD signal during task in the Tetris group at baseline $(p<.05$ FWE). Functional activations while playing Tetris before practice period.

Click here for file

[http://www.biomedcentral.com/content/supplementary/17560500-2-174-S1.doc]

\section{Additional file 2}

Brain areas of decreased BOLD signal during task in the Tetris group at baseline $(p<.05$ FWE). Functional deactivations while playing Tetris before practice period.

Click here for file

[http://www.biomedcentral.com/content/supplementary/17560500-2-174-S2.doc] 


\section{Additional file 3}

Brain areas of increased BOLD signal during task in the Tetris group at follow-up $(p<.05$ FWE). Functional activations while playing Tetris after practice period.

Click here for file

[http://www.biomedcentral.com/content/supplementary/17560500-2-174-S3.doc]

\section{Additional file 4}

Brain areas of decreased BOLD signal during task in the Tetris group at follow-up $(p<.05$ FWE). Functional deactivations while playing Tetris after practice period.

Click here for file

[http://www.biomedcentral.com/content/supplementary/17560500-2-174-S4.doc]

\section{Acknowledgements}

The authors thank Ranee Barrow (partial salary supported by the BPS funding) for invaluable help in data collection and Alan Evans for supporting cortical thickness analyses done at MNI.

\section{References}

I. Boyke J, Driemeyer J, Gaser C, Buechel C, May A: Training-induced brain structure changes in the elderly. Journal of Neuroscience 2008, 28(28):703।-7035.

2. Draganski B, Gaser C, Busch V, Schuierer G, Bogdahn U, May A: Neuroplasticity: changes in grey matter induced by training. Nature 2004, 427(6972):3। I-3 I2.

3. Draganski B, Gaser C, Kempermann G, Kuhn HG, Winkler J, Buchel C. May A: Temporal and Spatial Dynamics of Brain Structure Changes during Extensive Learning. J Neurosci 2006, 26(23):63|4-6317.

4. Driemeyer J, Boyke J, Gaser C, Buchel C, May A: Changes in gray matter induced by learning--revisited. PLOS ONE 2008, 3(7): 2669 .

5. Haier RJ, Siegel BV Jr, MacLachlan A, Soderling E, Lottenberg S, Buchsbaum MS: Regional glucose metabolic changes after learning a complex visuospatial/motor task: a positron emission tomographic study. Brain Res |992, 570(|-2): |34-| 43.

6. McNab F, Varrone A, Farde L, Jucaite A, Bystritsky P, Forssberg H, Klingberg T: Changes in cortical dopamine $\mathrm{DI}$ receptor binding associated with cognitive training. Science 2009, 323(59 15):800-802.

7. Goodyear BG, Douglas EA: Decreasing task-related brain activity over repeated functional MRI scans and sessions with no change in performance: implications for serial investigations. Exp Brain Res 2009, 192(2):231-239.

8. Little DM, Klein R, Shobat DM, McClure ED, Thulborn KR: Changing patterns of brain activation during category learning revealed by functional MRI. Brain Res Cogn Brain Res 2004, 22(I):84-93

9. Landau SM, Schumacher EH, Garavan H, Druzgal TJ, D'Esposito M: A functional MRI study of the influence of practice on component processes of working memory. Neuroimage 2004, 22(I):2II-22I.

10. Wu T, Kansaku K, Hallett M: How self-initiated memorized movements become automatic: a functional MRI study. I Neurophysiol 2004, 9 I(4): 1690-1698.

II. Karni A, Meyer G, Jezzard P, Adams MM, Turner R, Ungerleider LG: Functional MRI evidence for adult motor cortex plasticity during motor skill learning. Nature 1995, 377(6545): 155-I58.

12. Ilg R, Wohlschlager AM, Gaser C, Liebau Y, Dauner R, Woller A, Zimmer C, Zihl j, Muhlau M: Gray matter increase induced by practice correlates with task-specific activation: a combined functional and morphometric magnetic resonance imaging study. J Neurosci 2008, 28(16):4210-42I5.
13. Thomas AG, Marrett S, Saad ZS, Ruff DA, Martin A, Bandettini PA: Functional but not structural changes associated with learning: An exploration of longitudinal Voxel-Based Morphometry (VBM). Neuroimage 2009, 48(I): I |7- 25.

14. Alivisatos B, Petrides M: Functional activation of the human brain during mental rotation. Neuropsychologia 1997. 35(2): III-II8.

15. Cohen MS, Kosslyn SM, Breiter HC, DiGirolamo GJ, Thompson WL, Anderson AK, Bookheimer SY, Rosen BR, Belliveau JW: Changes in cortical activity during mental rotation - A mapping study using functional MRI. Brain 1996, I 19:89-100.

16. O'Boyle MW, Cunnington R, Silk TJ, Vaughan D, Jackson G, Syngeniotis A, Egan GF: Mathematically gifted male adolescents activate a unique brain network during mental rotation. Cognitive Brain Research 2005, 25(2):583-587.

17. Karama S, Ad-Dab'bagh Y, Haier RJ, Deary IJ, Lyttelton OC, Lepage $C$, Evans AC: Positive association between cognitive ability and cortical thickness in a representative US sample of healthy 6 to 18 year-olds. Intelligence 2009, 37:145-155.

18. Lerch JP, Evans AC: Cortical thickness analysis examined through power analysis and a population simulation. Neuroimage 2005, 24(1):163-173.

19. Cohen MS: Parametric analysis of fMRI data using linear systems methods. Neurolmage 1997, 6(2):93-103.

20. Talairach J, Tournoux P: Co-planar stereotaxic atlas of the human brain: a 3-dimensional proportional system, an approach to cerebral imaging. Stuttgart; New York: New York: G. Thieme; Thieme Medical Publishers; 1988.

2I. Lancaster JL, Tordesillas-Gutierrez D, Martinez M, Salinas F, Evans A Zilles K, Mazziotta JC, Fox PT: Bias between MNI and Talairach coordinates analyzed using the ICBM-I52 brain template. Hum Brain Mapp 2007, 28( I I): I I 94-I205.

22. Chumbley JR, Friston KJ: False discovery rate revisited: FDR and topological inference using Gaussian random fields. Neuroimage 2009, 44(I):62-70.

23. Genovese CR, Lazar NA, Nichols T: Thresholding of statistical maps in functional neuroimaging using the false discovery rate. Neuroimage 2002, 15(4):870-878.

24. Logothetis NK: What we can do and what we cannot do with fMRI. Nature 2008, 453(7/97):869-878.

25. Lau HC, Rogers RD, Haggard P, Passingham RE: Attention to intention. Science 2004, 303(566I): I208-1210.

26. Nakamura K, Sakai K, Hikosaka O: Neuronal activity in medial frontal cortex during learning of sequential procedures. Journal of Neurophysiology 1998, 80(5):267I-2687.

27. Nachev P, Rees G, Parton A, Kennard C, Husain M: Volition and conflict in human medial frontal cortex. Current Biology 2005 , I5(2): I 22- I 28

28. Olson IR, Plotzker A, Ezzyat Y: The Enigmatic temporal pole: a review of findings on social and emotional processing. Brain 2007, I30(Pt 7): |7|8-|73|

29. Markowitsch HJ: Which brain regions are critically involved in the retrieval of old episodic memory? Brain Res Brain Res Rev | 995, 2 I(2): | | 7- | 27

Publish with Bio Med Central and every scientist can read your work free of charge

"BioMed Central will be the most significant development for disseminating the results of biomedical research in our lifetime. "

Sir Paul Nurse, Cancer Research UK

Your research papers will be:

- available free of charge to the entire biomedical community

- peer reviewed and published immediately upon acceptance

- cited in PubMed and archived on PubMed Central

- yours - you keep the copyright 